\title{
A Fast Ray Space Transform for Wave Field Processing using Acoustic Arrays
}

\author{
Federico Borra, Mirco Pezzoli, Luca Comanducci, Alberto Bernardini \\ Fabio Antonacci, Stefano Tubaro, Augusto Sarti \\ Dipartimento di Elettronica, Informazione e Bioingegneria (DEIB), Politecnico di Milano \\ Piazza Leonardo Da Vinci 32, 20122 Milano, Italy \\ federico.borra@polimi.it,mirco.pezzoli@polimi.it, luca.comanducci@polimi.it, alberto.bernardini@polimi.it \\ fabio.antonacci@polimi.it, stefano.tubaro@polimi.it, augusto.sarti@polimi.it
}

\begin{abstract}
The importance of soundfield imaging techniques is expected to further increase in the next few years thanks to the ever-increasing availability of low-cost sensors such as MEMS microphones. When it comes to processing a relevant number of sensor signals, however, the computational load of space-time processing algorithms easily grows to unmanageable levels. The Ray Space Transform (RST) was recently introduced as a promising tool for soundfield analysis. Given the collection of signals captured by a uniform linear array of microphones, the RST allows us to collect and map the directional components of the acoustic field onto a domain called "ray space", where relevant acoustic objects are represented as linear patterns for advanced acoustic analysis and synthesis applications. So far the computational complexity of the RST linearly increases with the number of microphones. In order to alleviate this problem, in this paper we propose an alternative efficient implementation of the RST based on the Non Uniform Fast Fourier Transform.
\end{abstract}

Index Terms-Array Processing, Space-time Analysis, Soundfield Imaging, FFT

\section{INTRODUCTION}

The purpose of soundfield imaging is to describe acoustic scenes captured by arrays of microphones using a structured and unified representation. This enables the development of a wide range of soundfield analysis and processing applications [1]-[7]. In this context, the Ray Space Transform (RST) was recently developed as a promising framework for wavefield processing [8], that generalizes and formalizes the directional plenacoustic analysis introduced in [5], [9], [10] through the adoption of discrete Gabor frames [11]. The RST provides a decomposition of the acoustic field that relies on an overcomplete basis of wave field functions of local validity [8]. Such decomposition maps the directional components of the acoustic field onto a domain known as "ray space". In that domain, relevant acoustic objects, such as acoustic sources and reflectors, are represented as linear patterns (see Fig. 1 for an example showing how a point source in the geometric domain is mapped to a line in the ray space). More precisely, given a set of signals acquired by a Uniform Linear Array (ULA) of microphones, the RST employs a spatial sliding window in order to perform a "local" plane-wave decomposition of the recorded soundfield. This transformation has been exploited in many applications, among which source localization [12], source separation [13], soundfield reconstruction and manipulation [14].
So far the RST has been implemented as a matrix-vector multiplication [8], [15] whose computational cost linearly increases with the number of microphones. Recent advances in digital acoustic sensing technology (MEMS microphones) have made it possible to produce low-cost systems accommodating a large number of microphones, therefore faster algorithms for computing the RST would be desirable.

In this paper we show that the RST can be interpreted as a nonuniform Fourier transform and this can be exploited for developing an alternative highly-efficient implementation of the RST, based on the theory of Nonuniform Fast Fourier Transform (NUFFT) [16]-[22]. In line with most NUFFT implementations, the proposed algorithm involves two steps. The former requires the computation of an uniform oversampled discrete Fourier transform through a Fast Fourier Transform (FFT) algorithm. The latter consists of an interpolation process for deriving the RST coefficients. Like all NUFFT methods, this algorithm turns out to be characterized by a trade-off between accuracy and computational complexity depending on the oversampling factor and the interpolation order.

The rest of the paper is organized as follows. Section II provides a background on the RST according to [8]. Section III offers a detailed description of the proposed algorithm for the fast computation of the RST. Section IV validates the proposed algorithm through simulations and presents a comparison in terms of computational complexity with respect to the standard RST implementation. Section V draws the conclusions.

\section{RAY SPACE TRANSFORM}

The RST was first defined in [8] as a linear operator that maps the signals acquired by a ULA of microphones onto the ray space [5].

Let us consider an acoustic point source located in $\mathbf{r}^{\prime}$ and a ULA consisting of $L$ omnidirectional microphones, aligned on the $z$ axis between 0 and $2 q_{0}$ (see Fig. 1(a)). Denoting with $d$ the distance between two consecutive sensors, the position of the $l$ th array element is defined as $z_{l}=l d$ with $l=0, \ldots, L-$ 1. For the sake of convenience, the array geometry is here defined in a slightly different fashion with respect to [8] (the origin of the axis is shifted). 


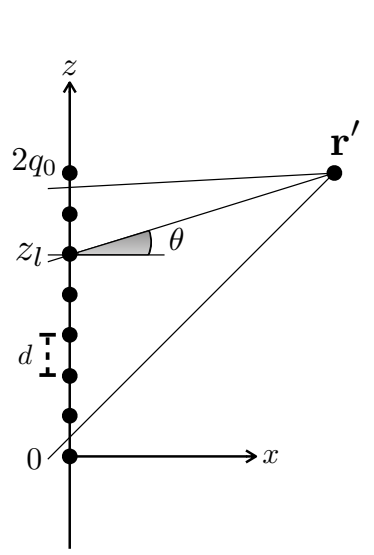

(a)

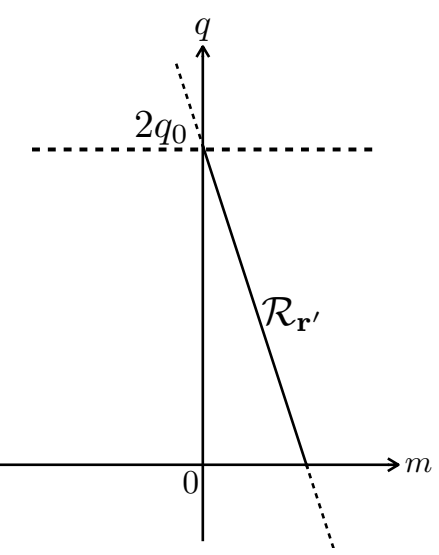

(b)
Fig. 1. (a) The ULA setup. (b) The representation $\mathcal{R}_{\mathbf{r}^{\prime}}$ of the point source in $\mathbf{r}^{\prime}$ in the ray space.

The $l$ th sensor signal, in the absence of additive noise, is defined in the frequency domain as

$$
p\left(z_{l}, \omega\right)=h\left(z_{l} \mid \mathbf{r}^{\prime}, \omega\right) \eta(\omega),
$$

where, $z_{l}$ is the microphone location, $\omega=2 \pi f$ is the angular frequency, $f>0$ is the temporal frequency and $h\left(z_{l} \mid \mathbf{r}^{\prime}, \omega\right)$ represents the transfer function of the source signal $\eta(\omega)$, from the source location $\mathbf{r}^{\prime}$ to the $l$ th microphone location.

The RST processes the array signals (1) performing a local plane-wave decomposition [23] where the direction $\theta$ of each local plane-wave component (acoustic ray) is parametrized according to the euclidean line equation

$$
z=m x+q,
$$

where $m=\tan (\theta)$ and $q$ are the parameters of the lines on which the rays lie on. The space identified by such parameters is known as ray space [5].

According to [8], the RST of the array data is

$$
[\mathbf{Z}]_{i, w}(\omega)=d \sum_{l=0}^{L-1} p\left(z_{l}, \omega\right) e^{\frac{-j k z_{l} m_{w}}{\sqrt{1+m_{w}^{2}}}} \psi_{i, l}^{*},
$$

where $j$ imaginary unit and $k=\omega / c$ with $c$ speed of sound. The indices $i=0, \ldots, I-1$ and $w=0, \ldots, W-1$ span the uniformly sampled ray space axes $q_{i}=i \bar{q}$ and $m_{w}=$ $(w-(W-1) / 2) \bar{m}$ defined by the sampling intervals $\bar{m}$ and $\bar{q}$. It is worth noting that by uniformly sampling the $m$ axis and the $q$ axis, acoustic sources and reflectors are mapped onto linear patterns in the ray space domain [5]. This feature can be exploited, for example, for developing sources and reflectors localization algorithms through pattern analysis techniques [5], [10], [24]. The term $\psi_{i, l}$ is the spatial window and a Gaussian window is commonly adopted, i.e.,

$$
\psi_{i, l}=e^{-\frac{\pi\left(z_{l}-q_{i}\right)^{2}}{\sigma^{2}}},
$$

where $\sigma$ controls the width of the Gaussian window centered in $q_{i}$. Considering a single spatial window i.e. fixing $i$, the
RST (3) corresponds to a local delay-and-sum beamforming operation on the array signals [25]. Notice that in (3), the phase shifts given by the $W$ spatial frequencies are expressed in terms of the ray space parametrization

$$
z \sin \left(\theta_{w}\right)=z \sin \left(\arctan \left(m_{w}\right)\right)=\frac{z m_{w}}{\sqrt{1+m_{w}^{2}}} .
$$

It is important to mention that [8] also introduces and defines the inverse transform of the RST, which allows us to losslessly recover the original audio signals from the soundfield image, thus confirming that the RST is, in fact, an effective and complete tool for soundfield processing purposes.

\section{Proposed Algorithm}

In this section we describe the proposed algorithm for a fast implementation of the RST. Let us define the nonuniform discrete Fourier transform of a signal $\bar{p}_{i, l}$ as follows [26]

$$
[\mathbf{F}]_{i, w}=\sum_{l=0}^{L-1} \bar{p}_{i, l} e^{-j \gamma_{w} l}
$$

where $\gamma_{w}, w=0, \ldots, W-1$ is a set of nonuniformly spaced frequency locations. In order to show that the RST can be interpreted as a nonuniform discrete Fourier transform, let us equate (3) to (6) as follows

$$
d \sum_{l=0}^{L-1} p\left(z_{l}, \omega\right) e^{\frac{-j k z_{l} m_{w}}{\sqrt{1+m_{w}^{2}}}} \psi_{i, l}^{*}=\sum_{l=0}^{L-1} \bar{p}_{i, l} e^{-j \gamma_{w} l} .
$$

The equality in (7) is verified under the following conditions

$$
\bar{p}_{i, l}=d p\left(z_{l}, \omega\right) \psi_{i, l}^{*}
$$

and

$$
\gamma_{w}=k \frac{m_{w} d}{\sqrt{1+m_{w}^{2}}} .
$$

Therefore, given (7) and the conditions in (8) and (9), for each frame $i=0, \ldots, I-1$, the RST can be rewritten in the form of a nonuniform discrete Fourier transform as

$$
[\mathbf{Z}]_{i, w}(\omega)=\sum_{l=0}^{L-1} \bar{p}_{i, l} e^{-j \gamma_{w} l} .
$$

A direct evaluation of (10) would require $\mathcal{O}(L W)$ operations for each frame $i=0, \ldots, I-1$. However, in the literature many algorithms have been proposed for a fast computation of the nonuniform discrete Fourier transform [16]-[22]. Most of them are based on a two-step approach.

The first step consists of the computation of an oversampled discrete Fourier transform by mean of a $N$-point Fast Fourier Transform:

$$
[\mathbf{Y}]_{i, n}=\sum_{l=0}^{L-1} s_{l} \bar{p}_{i, l} e^{-j \frac{2 \pi}{N} n l},
$$

where $n=0, \ldots, N-1$ with $N>L$ and $s_{l}$ are known as scaling factors and are usually designed to reduce the error that is introduced by the subsequent interpolation step [27]. The computation of $(11)$ requires $\mathcal{O}(N \log N)$ operations if implemented with an efficient algorithm [28]-[30]. As shown 


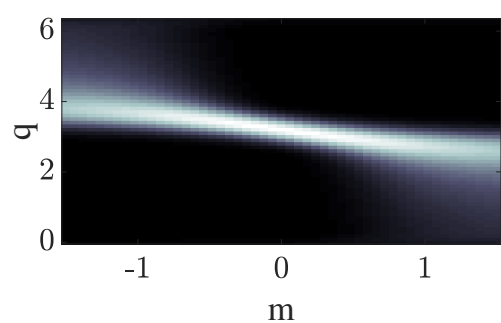

(a)

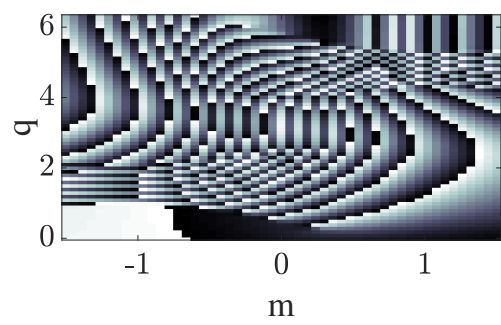

(d)

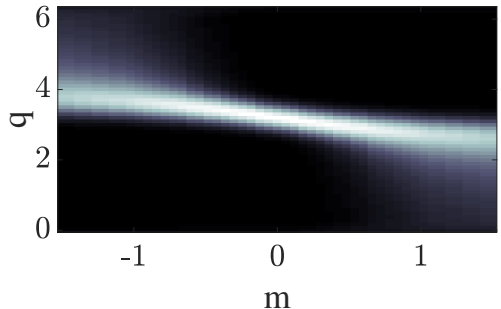

(b)

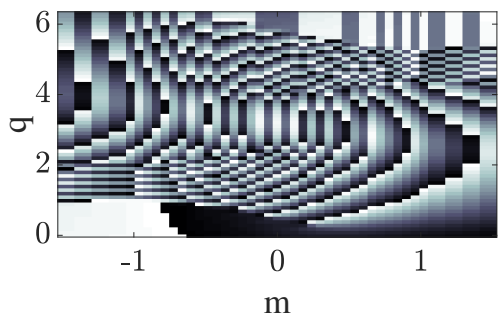

(e)

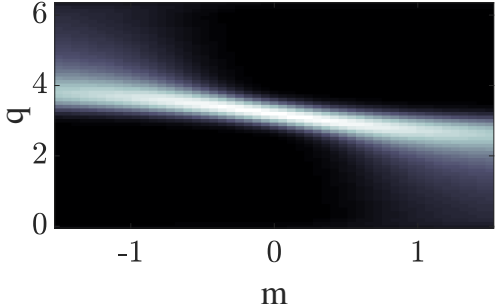

(c)

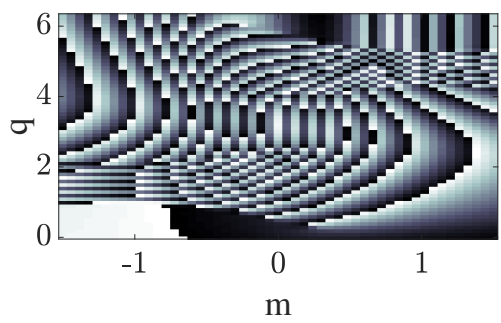

(f)

Fig. 2. (a) RST magnitude, (b) FRST magnitude, $B=1$, (c) FRST magnitude, $B=5$, (d) RST phase, (e) FRST phase, $B=1$, (f) FRST phase, $B=5$.

in [26], this cost can be further reduced to $\mathcal{O}(N \log L)$ if the ratio $N / L$ is an integer. In fact, in this case, one needs to perform $N / L L$-point FFTs [31].

The second step consists in an interpolation of the uniformly spaced frequency samples $[\mathbf{Y}]_{i, n}$ in order to obtain an approximation $[\widehat{\mathbf{Z}}]_{i, w}$ of $[\mathbf{Z}]_{i, w}$, i.e.

$$
[\widehat{\mathbf{Z}}]_{i, w}=\sum_{n=0}^{N-1} v_{w n}^{*}[\mathbf{Y}]_{i, n},
$$

where $v_{w n}^{*}$ are the interpolation coefficients. A direct evaluation of (12) would require $\mathcal{O}(W N)$ operations. However, usually the interpolation is accomplished by using only the $B$ nearest neighbors to $\gamma_{w}$ with $B \ll N$. More precisely, this can be written as

$$
[\widehat{\mathbf{Z}}]_{i, w}=\sum_{b=1}^{B} u_{w b}^{*}[\mathbf{Y}]_{i,\left\{n_{w}+b\right\}_{N}},
$$

where $\{\cdot\}_{N}$ is the modulo- $N$ operator and $n_{w}$ is defined as

$$
n_{w}=\left\{\begin{array}{lll}
\arg \min _{n}\left|\gamma_{w}-\frac{2 \pi}{N} n\right|-\frac{B+1}{2}, & B & \text { odd } \\
\max \left(n: \gamma_{w} \geq \frac{2 \pi}{N} n\right)-\frac{B}{2}, & B & \text { even. }
\end{array}\right.
$$

Various algorithms can be used to find both the interpolation coefficients $u_{w b}^{*}$ and the scaling factors $s_{l}$ as discussed in [16], [17], [27], [32]. In this paper we consider two possible approaches for the design of these parameters. The first one is the most straightforward and consists in approximating the value $[\widehat{\mathbf{Z}}]_{i, w}$ with the nearest neighbor. This means setting $B=1$ and $u_{w b}^{*}=1$ in (13) and (14) and $s_{l}=1$ in (11). The second approach is the one presented in [26], where the authors adopt a min-max criterion for optimizing the parameters of a Kaiser-Bessel interpolation kernel [33]. As discussed by the authors in [26], since in our case we need to compute multiple NUFFTs of the same size (one for each frame $i$ ), using the min-max approach with a Kaiser-Bessel interpolation kernel provides the highest accuracy among all the methods investigated in [26]. Moreover, such approach allows the reduction of the neighborhood size $B$ and, hence, the minimization of the computational complexity.

In the following, we will refer to the transformation in (13) as Fast Ray Space Transform (FRST).

\section{RESUlTS}

In this section we present some simulation results in order to validate both the efficiency and the accuracy of the FRST. In the following, whenever $B=1$ we refer to the nearest neighbor criterion, in all other cases we refer to the min-max one.

An implementation of both the RST and the FRST is provided online ${ }^{1}$.

\section{A. Simulations}

The setup adopted for the simulations consists of an array of $L=64$ microphones, comprised between $z_{1}=0 \mathrm{~m}$ and $z_{L}=$ $6.3 \mathrm{~m}$, thus the spacing between consecutive microphones is $d=0.1 \mathrm{~m}$. The ray space is sampled using $W=51$ and $I=64$ points on the $m$ axis and the $q$ axis, respectively, with sampling intervals $\bar{m}=0.06$ and $\bar{q}=0.1$.

Given the previously described setup, let us assume that the source in is $\mathbf{r}^{\prime}=[0.5 \mathrm{~m}, 3.2 \mathrm{~m}]^{T}$ is emitting a sinusoidal signal with temporal frequency $f=1000 \mathrm{~Hz}$. Fig. 2 shows both the magnitude and the phase of the output obtained from the RST and FRST. Fig. 2(a) and Fig. 2(d) refer to the RST,

\footnotetext{
${ }^{1}$ https://github.com/polimi-ispl/ray-space-transform
} 


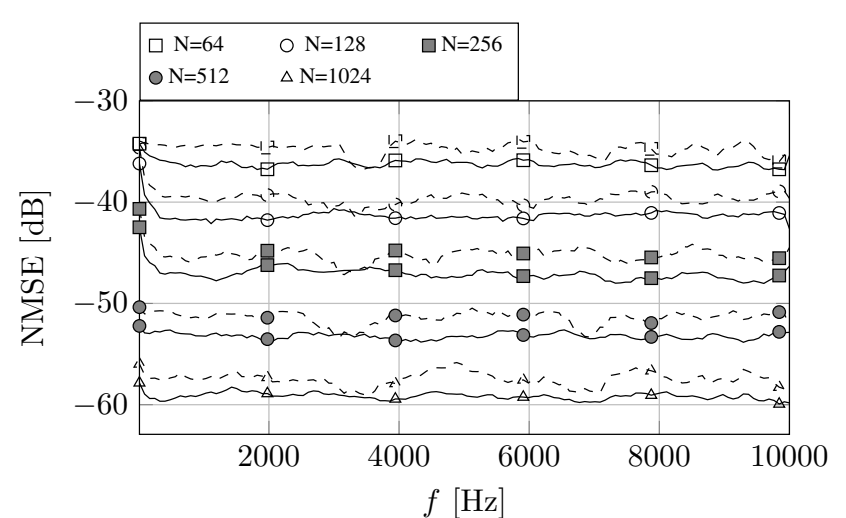

(a)

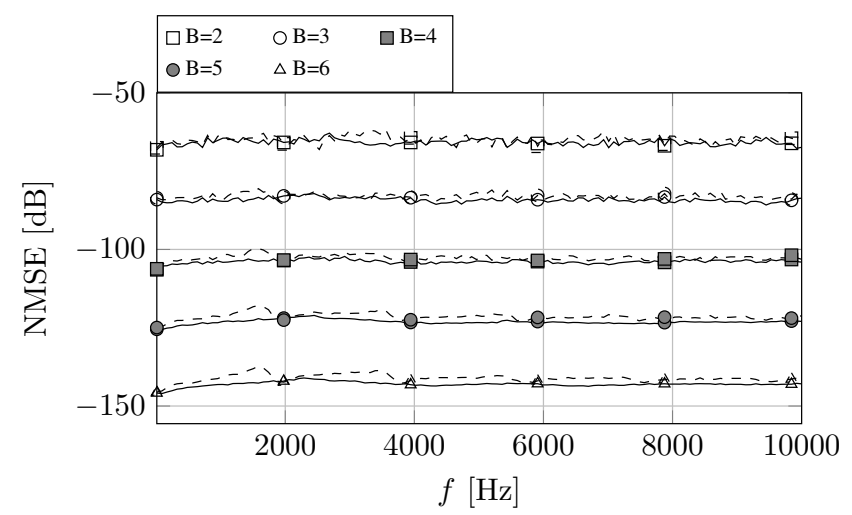

(b)

Fig. 3. NMSE as a function of temporal frequency when (a) varying the FFT length $N$ and (b) varying the $B$ nearest neighbors used for the interpolation. Solid lines refer to the source in $\mathbf{r}_{1}^{\prime}$ while dashed lines refer to the source in $\mathbf{r}_{2}^{\prime}$.

Fig. 2(b) and Fig. 2(e) to the FRST in the case where $B=1$, while Fig. 2(c) and Fig. 2(f) in the case where $B=5$. Clearly, the results obtained with an higher interpolation order $B$ are characterized by an higher accuracy. This fact is particularly evident when one considers the phase behavior.

In order to objectively measure the accuracy of the FRST, we define the Normalized Mean Squared Error as

$\mathrm{NMSE}=10 \log _{10}\left(\frac{1}{W I} \sum_{i=0}^{I-1} \sum_{w=0}^{W-1} \frac{\left|[\mathbf{Z}]_{i, w}(\omega)-[\widehat{\mathbf{Z}}]_{i, w}(\omega)\right|^{2}}{\left|[\mathbf{Z}]_{i, w}(\omega)\right|^{2}}\right)$.

In Fig. 3 we show the NMSE as a function of $f$ in a range between $10 \mathrm{~Hz}$ and $10 \mathrm{kHz}$. Without recurring to a complete evaluation for all possible positions of the sources, from preliminary results we selected the two most significant positions. In particular, the first source is placed in $\mathbf{r}_{1}^{\prime}=[5 \mathrm{~m}, 3.15 \mathrm{~m}]^{T}$ and the second in $\mathbf{r}_{2}^{\prime}=[0.2 \mathrm{~m}, 10 \mathrm{~m}]^{T}$. Hence, the former is in the end fire while the latter in the broadside with respect to the array. Fig. 3(a) shows the NMSE as a function of the frequency for different oversampling factors $N$ with $B=1$. The solid lines are referred to the source in $\mathbf{r}_{1}^{\prime}$, while the dashed lines to the source in $\mathbf{r}_{2}^{\prime}$. As expected higher $N$ values

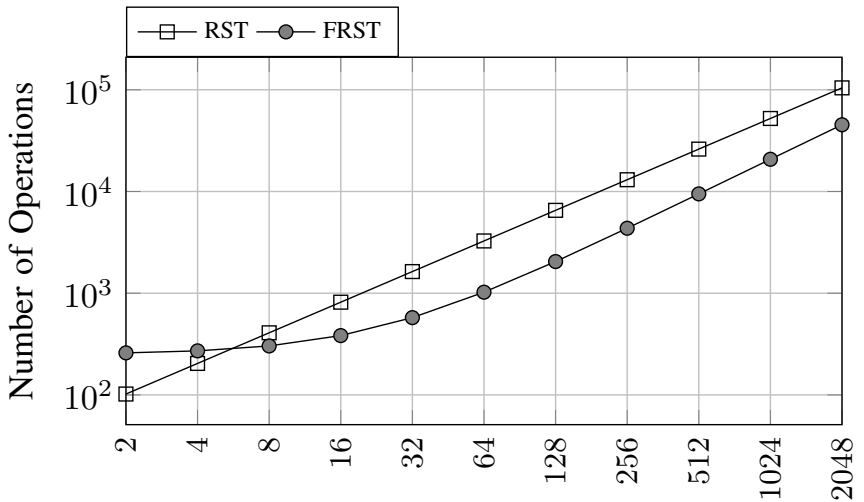

$L$

(a)

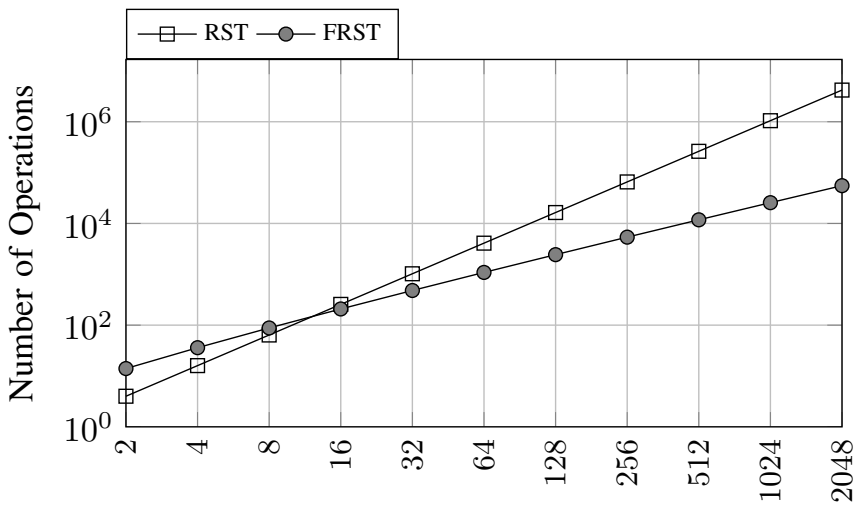

$L$

(b)

Fig. 4. Number of operations as a function of number of microphones $L$. (a) considers $W=51$ spatial frequencies, while (b) $W=L$. The $\mathrm{x}$ and y axis are on a logarithmic scale.

correspond to a smaller NMSE for both the sources, but a small differences can be observed between the two positions. In Fig. 3(b), instead, we keep $N=128$ and vary $B$. As expected, choosing a higher interpolation factor $B$ leads to better results in terms of NMSE. If we compare Fig. 3(b) with the curve corresponding to $N=128$ in Fig. 3(a) we can see that, even for $B=2$, we achieve higher accuracy. Moreover, the differences between the two sources considerably decrease. This suggests that choosing an interpolation order $B>1$ leads to more accurate results with respect to the nearest neighbor approach.

\section{B. Computational Complexity}

In the following we provide an analysis of the computational complexity of the FRST and the RST based on the considerations introduced in Sec. III. For a given frame $i$, the FRST requires $\mathcal{O}(N \log N+W B)$ operations when the ratio $N / L$ is not an integer and $\mathcal{O}(N \log L+W B)$ operations when the ratio $N / L$ is integer. On the other hand, the RST requires $\mathcal{O}(L W)$ operations. 
Fig. 4 shows the number of needed operations as a function of the number of microphones $L$. In particular, $L$ assumes the values in the set $\{2,4,8,16,32,64,128,256,512,1024,2048\}$ and, since we fix $N=2 L$, the ratio $N / L$ is an integer. In Fig. 4(a) we keep $W=51$ fixed for each value of $L$, while in Fig. 4(b) we choose $W=L$. In both cases we set $B=5$. By inspecting both Fig. 4(a) and Fig. 4(b) it is clear how our implementation of the RST requires less operations, especially as the number of microphones $L$ increases.

\section{CONClusions And Future Works}

In this paper we proposed a fast algorithm for the computation of the RST. We showed that the RST can be interpreted as a nonuniform Fourier transform and, based on that, we used the theory of NUFFT for developing our algorithm. The results that we presented show that the proposed method improves scalability with respect to the traditional RST implementation in terms of computational complexity, while introducing a negligible approximation error. We are currently working on the extension of the proposed approach to nonuniform linear array geometries and an implementation of a fast version of the inverse RST.

\section{REFERENCES}

[1] B. N. Gover, J. G. Ryan, and M. R. Stinson, "Microphone array measurement system for analysis of directional and spatial variations of sound field," J. Acoust. Soc. Am., vol. 112, no. 5, pp. 1980-1991, Nov. 2002.

[2] F. Pinto and M. Vetterli, "Applications of short space-time Fourier analysis in digital acoustics," in Proc. IEEE Int. Conf. on Acoustics, Speech, and Signal Process. (ICASSP), 2011.

[3] D. Khaykin and B. Rafaely, "Acoustic analysis by spherical microphone array processing of room impulse responses," J. Acoust. Soc. Am., vol. 132, no. 1, pp. 261-270, July 2012.

[4] N. Epain and C. T. Jin, "Super-resolution sound field imaging with subspace pre-processing," in Proc. IEEE Int. Conf. on Acoustics, Speech, and Signal Process. (ICASSP), 2013.

[5] D. Markovic, F. Antonacci, A. Sarti, and S. Tubaro, "Soundfield imaging in the ray space," IEEE Transactions on Audio, Speech, and Language Processing, vol. 21, no. 12, pp. 2493-2505, 2013.

[6] M. Samarawickrama, N. Epain, and C. Jin, "Super-resolution acoustic imaging using non-uniform spatial dictionaries," in Proc. IEEE Int. Conf. on Audio, Language and Image Process., 2014.

[7] L. Bianchi, M. Verdi, F. Antonacci, A. Sarti, and S. Tubaro, "High resolution imaging of acoustic reflections with spherical microphone array," in Proc. IEEE Workshop on Applications of Signal Process. to Audio and Acoustics (WASPAA), 2015.

[8] L. Bianchi, F. Antonacci, A. Sarti, and S. Tubaro, "The ray space transform: A new framework for wave field processing," IEEE Transactions on Signal Processing, vol. 64, no. 21, pp. 5696-5706, Nov. 2016.

[9] D. Markovic, G. Sandrini, F. Antonacci, A. Sarti, and S. Tubaro, "Plenacoustic imaging in the ray space," in IWAENC 2012; International Workshop on Acoustic Signal Enhancement, Sep. 2012, pp. 1-4.

[10] D. Markovic, F. Antonacci, A. Sarti, and S. Tubaro, "Multiview Soundfield Imaging in the Projective Ray Space," IEEE/ACM Transactions on Audio, Speech, and Language Processing, vol. 23, no. 6, pp. 1054-1067, Jun. 2015. [Online]. Available: http://ieeexplore. ieee.org/document/7076611/

[12] L. Comanducci, F. Borra, P. Bestagini, F. Antonacci, A. Sarti, and S. Tubaro, "Ray space transform interpolation with convolutional autoencoder," in 2018 16th International Workshop on Acoustic Signal Enhancement (IWAENC). IEEE, 2018, pp. 261-265.
[11] S. Qian and D. Chen, "Discrete Gabor transform," IEEE Transactions on Signal Processing, vol. 41, no. 7, pp. 2429-2438, 1993.

[13] F. Borra, F. Antonacci, A. Sarti, and S. Tubaro, "Extraction of acoustic sources for multiple arrays based on the ray space transform," in 2017 Hands-Free Speech Communications and Microphone Arrays (HSCMA). IEEE, 2017, pp. 146-150.

[14] M. Pezzoli, F. Borra, F. Antonacci, A. Sarti, and S. Tubaro, "Estimation of the sound field at arbitrary positions in distributed microphone networks based on distributed ray space transform," in 2018 IEEE International Conference on Acoustics, Speech and Signal Processing (ICASSP). IEEE, 2018, pp. 186-190.

[15] L. Bianchi, V. Baldini, D. Marković, F. Antonacci, A. Sarti, and S. Tubaro, "A linear operator for the computation of soundfield maps," in Proc. IEEE Int. Conf. on Acoustics, Speech, and Signal Process. (ICASSP), 2016.

[16] A. Dutt and V. Rokhlin, "Fast Fourier transforms for nonequispaced data," SIAM Journal on Scientific computing, vol. 14, no. 6, pp. 13681393, 1993.

[17] _ _ "Fast Fourier transforms for nonequispaced data, ii," Applied and Computational Harmonic Analysis, vol. 2, no. 1, pp. 85-100, 1995.

[18] Q. H. Liu and N. Nguyen, "An accurate algorithm for nonuniform fast Fourier transforms (NUFFT's)," IEEE Microwave and guided wave letters, vol. 8, no. 1, pp. 18-20, 1998.

[19] G. Steidl, "A note on fast Fourier transforms for nonequispaced grids," Advances in computational mathematics, vol. 9, no. 3-4, pp. 337-352, 1998.

[20] A. F. Ware, "Fast approximate Fourier transforms for irregularly spaced data," SIAM review, vol. 40, no. 4, pp. 838-856, 1998.

[21] A. J. W. Duijndam and M. A. Schonewille, "Nonuniform fast Fourier transform," Geophysics, vol. 64, no. 2, pp. 539-551, 1999.

[22] D. Potts, G. Steidl, and M. Tasche, Fast Fourier transforms for nonequispaced data: a tutorial. Boston, MA: Birkhäuser Boston, 2001, pp. 247-270. [Online]. Available: https://doi.org/10.1007/ 978-1-4612-0143-4_12

[23] E. G. Williams, Fourier acoustics: sound radiation and nearfield acoustic holography. London, UK: Academic Press, 1999.

[24] F. Borra, F. Antonacci, A. Sarti, and S. Tubaro, "Localization of acoustic sources in the ray space for distributed microphone sensors," in 2017 IEEE Workshop on Applications of Signal Processing to Audio and Acoustics (WASPAA). IEEE, 2017, pp. 170-174.

[25] H. L. Van Trees, Optimum array processing: part IV of detection, estimation and modulation theory. Wiley Online Library, 2002, vol. 1.

[26] J. A. Fessler and B. P. Sutton, "Nonuniform fast Fourier transforms using min-max interpolation," IEEE transactions on signal processing, vol. 51 , no. 2 , pp. 560-574, 2003.

[27] N. Nguyen and Q. H. Liu, "The regular Fourier matrices and nonuniform fast Fourier transforms," SIAM Journal on Scientific Computing, vol. 21, no. 1, pp. 283-293, 1999.

[28] J. W. Cooley and J. W. Tukey, "An algorithm for the machine calculation of complex Fourier series," Mathematics of Computation, vol. 19, no. 90, pp. 297-301, 1965. [Online]. Available: http: //www.jstor.org/stable/2003354

[29] P. Duhamel and M. Vetterli, "Fast Fourier transforms: A tutorial review and a state of the art," Signal Processing (Elsevier), vol. 19, no. 4, pp. 259-299, 1990. [Online]. Available: http: //infoscience.epfl.ch/record/59946

[30] C. Van Loan, Computational frameworks for the fast Fourier transform. Siam, 1992, vol. 10.

[31] T. Sreenivas and P. Rao, "FFT algorithm for both input and output pruning," IEEE Transactions on Acoustics, Speech, and Signal Processing, vol. 27 , no. 3, pp. 291-292, June 1979.

[32] M. D. Macleod, "Fast nearly ML estimation of the parameters of real or complex single tones or resolved multiple tones," IEEE Transactions on Signal Processing, vol. 46, no. 1, pp. 141-148, Jan 1998.

[33] A. Nuttall, "Some windows with very good sidelobe behavior," IEEE Transactions on Acoustics, Speech, and Signal Processing, vol. 29, no. 1, pp. 84-91, February 1981 